1 Hacettepe Journal of Mathematics and Statistics

h Volume 47 (3) (2018), 615-623

\title{
A fixed point approach to the stability of a nonlinear volterra integrodifferential equation with delay
}

\author{
Rahim Shah* and Akbar Zada ${ }^{\dagger}$
}

\begin{abstract}
By using a fixed point method, we prove the Hyers-Ulam-Rassias stability and the Hyers-Ulam stability of a nonlinear Volterra integrodifferential equation with delay. Two examples are presented to support the usability of our results.
\end{abstract}

Keywords: Hyers-Ulam-Rassias stability, Hyers-Ulam stability, Volterra integrodifferential equation with delay, fixed point approach.

2000 AMS Classification: 47H10, 45J05, 45M10.

Received : 05.01.2017 Accepted : 20.03.2017 Doi : 10.15672/HJMS.2017.467

\section{Introduction}

In 1940, Ulam posed the following problem related to the stability of functional equations: "Under what conditions does there exist an additive mapping near an approximately additive mapping?" see for more detail [15]. One year later, Hyers [8] gave an answer to the problem of Ulam for the case of functional equation for homomorphism between the Banach spaces. In 1978, Rassias [13] proved the existence of unique linear mapping near approximate additive mapping that provides generalization of the Hyers result. Jung [9] applied the fixed point method to the investigation of Volterra integral equation by using the idea of Cadariu and Radu in [2]. S. M. Jung proved that if a continuous function $v: I \rightarrow \mathbb{C}$ is such that

$$
\left|v(t)-\int_{c}^{t} G(\xi, v(\xi)) d \xi\right| \leq \phi(t)
$$

*Department of Mathematics, University of Peshawar, Peshawar 25000, Pakistan Email : safeer_rahim@yahoo.com

${ }^{\dagger}$ Department of Mathematics, University of Peshawar, Peshawar 25000, Pakistan

Email : zadababo@yahoo.com, akbarzada@uop.edu.pk 
for all $t \in I$, then there exists a unique continuous function $v_{0}: I \rightarrow \mathbb{C}$ and a constant $K>0$ such that

$$
v_{0}(t)=\int_{c}^{t} G\left(\xi, v_{0}(\xi)\right) d \xi \quad \text { and } \quad d\left(v(t), v_{0}(t)\right) \leq L \phi(t),
$$

for all $t \in I$, it is important to obtain a precise $L$ because it is clear that $L$ will lead us to the error between the actual solution $v_{0}(t)$ and the approximate solution $v(t)$. In 2013, Jung et al. proved that if $g: I \rightarrow \mathbb{R}, h: I \rightarrow \mathbb{R}, G: I \rightarrow \mathbb{R}$ and $\phi: I \rightarrow \mathbb{R}$ are sufficiently smooth functions and if a continuously differentiable function $v: I \rightarrow \mathbb{R}$ satisfies the perturbed Volterra integrodifferential equation

$$
\left|v^{\prime}(t)+g(t) v(t)+h(t)+\int_{c}^{t} K(t, \eta) v(\eta) d \eta\right| \leq \phi(t),
$$

for some $t \in I$, then there exists a unique solution $v_{0}: I \rightarrow \mathbb{R}$ of the Volterra integrodifferential equation

$$
v^{\prime}(t)+g(t) v(t)+h(t)+\int_{c}^{t} K(t, \eta) v(\eta) d \eta=0
$$

such that

$$
d\left(v(t), v_{0}(t)\right) \leq \exp \left\{-\int_{c}^{t} g(\eta) d \eta\right\} \int_{t}^{b} \phi(\varsigma) \exp \left\{\int_{c}^{\varsigma} g(\eta) d \eta\right\} d \varsigma,
$$

for all $t \in I$. If the reader wishes more details, we recommend $[1,3,4,6,7,12,14,16]$.

The main purpose of the paper is to investigate the Hyers-Ulam-Rassias stability and the Hyers-Ulam stability of following nonlinear Volterra integrodifferential equation with delay:

$$
v^{\prime}(t)=g(t, v(t), v(\alpha(t)))+\int_{0}^{t} k(t, s, v(s), v(\alpha(s))) d s
$$

for all $t \in I=[0, T]$, where the function $g(t, v(t), v(\alpha(t)))$ is continuous function with respect to variables $t$ and $v$ on $I \times \mathbb{R} \times \mathbb{R}, k(t, s, v(t), v(\alpha(t)))$ is continuous with respect to variables $t, s$ and $v$ on $I \times I \times \mathbb{R} \times \mathbb{R}, \beta$ is any constant and $\alpha:[0, T] \rightarrow[0, T]$ is a continuous delay function with $\alpha(t) \leq t$.

1.1. Definition. If for each continuously differentiable function $v(t)$ satisfying

$$
\left|v^{\prime}(t)-g(t, v(t), v(\alpha(t)))-\int_{0}^{t} k(t, s, v(s), v(\alpha(s))) d s\right| \leq \phi(t),
$$

for some $\phi:[0, T] \rightarrow(0, \infty)$, there exists a solution $v_{0}(t)$ of the Volterra integrodifferential equations with delay (1.1) and a constant $K>0$ (independent of $v(t)$ and $v_{0}(t)$ ) with

$$
\left|v(t)-v_{0}(t)\right| \leq K \phi(t),
$$

for all $t \in I$, then we can say that the equation (1.1) is Hyers-Ulam-Rassias stable on $I$. If $\phi(t)$ is constant function then we say that the equation (1.1) has Hyers-Ulam stability on $I$.

For a nonempty set $Y$, the generalized metric on $Y$ is defined as follow:

1.2. Definition. A function $d: Y \times Y \rightarrow[0, \infty]$ is called a generalized metric on $Y$ if and only if for all $u, v, w \in Y d$ satisfies the following conditions:

(1) $d(u, v)=0$ if and only if $u=v$.

(2) $d(u, v)=d(v, u)$.

(3) $d(u, w) \leq d(u, v)+d(v, w)$.

Now, we are going to introduce one of the most crucial result of fixed point theory that will play an important role in proving our main results. 
1.3. Theorem. ([5]) Let $(Y, d)$ be a generalized complete metric space. Assume that $\Theta: Y \rightarrow Y$ is a strictly contractive operator with $L<1$ as Lipschitz constant. If there exists a nonnegative integer $k$ such that $d\left(\Theta^{k+1} u, \Theta^{k} u\right)<\infty$ for some $u \in Y$, then the following conditions are true:

- The sequence $\Theta^{n} u$ converges to a fixed point $u^{*}$ of $\Theta$;

- $u^{*}$ is the unique fixed point of $\Theta$ in

$$
Y^{*}=\left\{v \in Y \mid d\left(\Theta^{k} u, v\right)<\infty\right\}
$$

- If $v \in Y^{*}$, then

$$
d\left(v, u^{*}\right) \leq \frac{1}{1-L} d(\Theta v, v)
$$

\section{Hyers-Ulam-Rassias stability}

In this section, we prove the Hyers-Ulam-Rassias stability of the nonlinear Volterra integrodifferential equation with delay (1.1).

2.1. Theorem. Let $I=[0, T]$ be a closed and bounded interval for a given $T>0$ and let $N, L_{g}$ and $L_{k}$ be nonnegative constants with $0<N L_{g}+N^{2} L_{k}<1$. Assume that $g: I \times \mathbb{R} \times \mathbb{R} \rightarrow \mathbb{R}$ is a continuous function that satisfies a Lipschitz condition

$$
\left|g\left(t, v_{1}, v_{1}(\alpha(t))\right)-g\left(t, v_{2}, v_{2}(\alpha(t))\right)\right| \leq L_{g}\left|v_{1}-v_{2}\right|,
$$

for all $t \in I$ and $v_{1}, v_{2} \in \mathbb{R}$. Let $k: I \times I \times \mathbb{R} \times \mathbb{R} \rightarrow \mathbb{R}$ be a continuous function which satisfies a Lipchitz condition

$$
\mid k\left(t, s, v_{1}, v_{1}(\alpha(s))\right)-k\left(t, s, v_{2}, v_{2}(\alpha(s))\left|\leq L_{k}\right| v_{1}-v_{2} \mid,\right.
$$

for all $t, s \in I, v_{1}, v_{2} \in \mathbb{R}$. If $v: I \rightarrow \mathbb{R}$ a continuously differentiable function satisfies

$$
\left|v^{\prime}(t)-g(t, v(t), v(\alpha(t)))-\int_{0}^{t} k(t, s, v(s), v(\alpha(s))) d s\right| \leq \phi(t),
$$

for all $t \in I$, where $\phi: I \rightarrow(0, \infty)$ is a continuous function with

$$
\int_{0}^{t} \phi(\zeta) d \zeta \leq N \phi(t)
$$

for all $t \in I$, then there exists a unique continuous function $v_{0}: I \rightarrow \mathbb{R}$ such that

$$
v_{0}(t)=\int_{0}^{t} g\left(\zeta, v_{0}(\zeta), v_{0}(\alpha(\zeta))\right) d \zeta+\int_{0}^{t} \int_{0}^{s} k\left(t, \zeta, v_{0}(\zeta), v_{0}(\alpha(\zeta)) d \zeta d s\right.
$$

and

$$
\left|v(t)-v_{0}(t)\right| \leq \frac{N}{1-\left(N L_{g}+N^{2} L_{k}\right)} \phi(t)
$$

for all $t \in I$.

Proof. Let $Y$ be the set of all real valued continuous functions on closed and bounded interval $I$. For all $u, w \in Y$, we set

$$
d(u, w)=\inf \{K \in[0, \infty]:|u(t)-w(t)| \leq K \phi(t), \text { for all } t \in I\} .
$$

The metric space $(Y, d)$ is a complete generalized metric space, see [10]. Consider the operator $\Theta: Y \rightarrow Y$ defined by

$$
(\Theta u)(t)=v(0)+\int_{0}^{t} g(\zeta, u(\zeta), u(\alpha(\zeta))) d \zeta+\int_{0}^{t} \int_{0}^{s} k(t, \zeta, u(\zeta), u(\alpha(\zeta))) d \zeta d s
$$


for all $t \in I$. We show that the operator $\Theta$ is strictly contractive. Let $K_{u w} \in[0, \infty]$ be a constant with $d(u, w) \leq K_{u w}$ for $u, w \in Y$. By (2.7), we can write

$$
|u(t)-w(t)| \leq K_{u w} \phi(t) \text { for all } t \in I \text {. }
$$

From inequalities (2.1)), (2.2), (2.4), (2.8) and (2.9) it follows that for all $t \in I$ we have

$$
\begin{aligned}
|(\Theta u)(t)-(\Theta w)(t)| & =\mid \int_{0}^{t}\{g(\zeta, u(\zeta), u(\alpha(\zeta)))-g(\zeta, w(\zeta), w(\alpha(\zeta)))\} d \zeta \\
& +\int_{0}^{t} \int_{0}^{s}\{k(t, \zeta, u(\zeta), u(\alpha(\zeta)))-k(t, \zeta, w(\zeta), w(\alpha(\zeta)))\} d \zeta d s \mid \\
& \leq \int_{0}^{t}|g(\zeta, u(\zeta), u(\alpha(\zeta)))-g(\zeta, w(\zeta), w(\alpha(\zeta)))| d \zeta \\
& +\int_{0}^{t} \int_{0}^{s}|k(t, \zeta, u(\zeta), u(\alpha(\zeta)))-k(t, \zeta, w(\zeta), w(\alpha(\zeta)))| d \zeta d s \\
& \leq L_{g} \int_{0}^{t}|u(\zeta)-w(\zeta)| d \zeta+L_{h} \int_{0}^{t} \int_{0}^{s}|u(\zeta)-w(\zeta)| d \zeta d s \\
& \leq L_{g} K_{u w} \int_{0}^{t} \phi(\zeta) d \zeta+L_{k} K_{u w} \int_{0}^{t} \int_{0}^{s} \phi(\zeta) d \zeta d s \\
& \leq K_{u w} \phi(t)\left(N L_{g}+N^{2} L_{k}\right),
\end{aligned}
$$

i.e. $d(\Theta u, \Theta w) \leq K_{u w} \phi(t)\left(N L_{g}+N^{2} L_{k}\right)$. Hence, we may conclude that $d(\Theta u, \Theta w) \leq$ $\left(N L_{g}+N^{2} L_{k}\right) d(u, w)$ for any $u, w \in Y$, where $0<N L_{g}+N^{2} L_{k}<1$.

It follows from (2.8) that for any arbitrary $w_{0} \in Y$, there exists a constant $K \in[0, \infty]$ with

$$
\begin{aligned}
\left|\Theta w_{0}(t)-w_{0}(t)\right| & =\mid v(0)+\int_{0}^{t} g\left(\zeta, w_{0}(\zeta), w_{0}(\alpha(\zeta))\right) d \zeta \\
& +\int_{0}^{t} \int_{0}^{s} k\left(t, \zeta, u_{0}(\zeta), u_{0}(\alpha(\zeta))\right) d \zeta d s-w_{0} \mid \\
& \leq K \phi(t), \text { for all } t \in I .
\end{aligned}
$$

Since $g\left(\zeta, w_{0}(\zeta), w_{0}(\alpha(\zeta))\right), k\left(t, \zeta, u_{0}(\zeta), u_{0}(\alpha(\zeta))\right)$ and $w_{0}$ are bounded and $\min _{t \in I} \phi(t)>$ 0 . Thus, (2.7) implies that

$$
d\left(\Theta w_{0}, w_{0}\right)<\infty .
$$

So, according to Theorem 1.3, there exists a continuous function $v_{0}: I \rightarrow \mathbb{R}$ in a way that $\Theta^{n} w_{0} \rightarrow v_{0}$ in $(Y, d)$ and $\Theta v_{0}=v_{0}$, i.e., $v_{0}$ satisfies (2.5) for all $t \in I$. Since we know that $w$ and $w_{0}$ are bounded on closed interval $I$ for any $w \in Y$ and $\min _{t \in I} \phi(t)>0$, then there exists a constant $K_{w} \in[0, \infty]$ such that

$$
d\left(w_{0}(t), w(t)\right) \leq K_{w} \phi(t)
$$

for any $t \in I$. We have $\left|w_{0}(t)-w(t)\right|<\infty$ for any $w \in Y$. Therefore, we get that $\left\{w \in Y \mid d\left(w_{0}, w\right)<\infty\right\}$ is equal to $Y$. From Theorem 1.3, we conclude that $v_{0}$, given by (2.5), is the unique continuous function. Again from (2.3), we get

$$
-\phi(t) \leq v^{\prime}(t)-g(t, v(t), v(\alpha(t)))-\int_{0}^{t} k(t, s, v(s), v(\alpha(s))) d s \leq \phi(t)
$$

for all $t \in I$. By integrating each term of inequality (2.10) from 0 to $t$, we get

$$
\left|v(t)-v(0)-\int_{0}^{t} g(\zeta, v(\zeta), v(\alpha(\zeta))) d \zeta-\int_{0}^{t} \int_{0}^{s} k(t, \zeta, v(\zeta), v(\alpha(\zeta))) d \zeta d s\right|
$$




$$
\leq \int_{0}^{t} \phi(\zeta) d \zeta
$$

for all $t \in I$. From (2.4) and (2.8), we get

$$
|v(t)-(\Theta v)(t)| \leq \int_{0}^{t} \phi(\zeta) d \zeta \leq N \phi(t)
$$

for all $t \in I$, which implies that $d(v, \Theta v) \leq N$. Next by making the use of Theorem 1.3 and inequality (2.11), we conclude that

$$
d\left(v, v_{0}\right) \leq \frac{1}{1-\left(N L_{g}+N^{2} L_{k}\right)} d(\Theta v, v) \leq \frac{N}{1-\left(N L_{g}+N^{2} L_{k}\right)} .
$$

Consequently, this yields the inequality (2.6) for all $t \in I$.

In the above Theorem 2.1 we examined the Hyers-Ulam-Rassias stability of (1.1) on a closed and bounded interval. Now, we are going to show that Theorem 2.1 is also valid for the case of unbounded interval.

2.2. Theorem. Suppose that $I$ denote either $\mathbb{R}$ or $[0, \infty)$ or $(-\infty, T]$ for a given nonnegative real number $T$. Let $L_{g}, L_{k}$ and $N$ be positive constants with $0<N L_{g}+N^{2} L_{k}<1$ and $\alpha: I \rightarrow \mathbb{R}$ be a continuous delay function such that $\alpha(t) \leq t$ for all $t \in I$. Assume that $g: I \times \mathbb{R} \times \mathbb{R} \rightarrow \mathbb{R}$ is a continuous function satisfying condition (2.1) for all $t \in I$, $v_{1}, v_{2} \in \mathbb{R}$. If a continuously differentiable function $v: I \rightarrow \mathbb{R}$ satisfies inequality (2.3) for all $t \in I$, where $\phi: I \rightarrow(0, \infty)$ is a continuous function satisfying condition (2.4) for all $t \in I$, then there exists a unique continuous function $v_{0}: I \rightarrow \mathbb{R}$ which satisfies (2.5) and (2.6) for each $t \in I$.

Proof. First we assume that $I=\mathbb{R}$ and we are going to show that $v_{0}$ is a continuous function. For any $n \in \mathbb{N}$, we define the interval $I_{n}=[-n, n]$. In accordance with Theorem 2.1 , there exists a unique continuous function $v_{n}: I_{n} \rightarrow \mathbb{R}$ in such a way that

$$
v_{n}(t)=v(0)+\int_{0}^{t} g\left(\zeta, v_{n}(\zeta), v_{n}(\alpha(\zeta))\right) d \zeta+\int_{0}^{t} \int_{0}^{s} k\left(t, \zeta, v_{n}(\zeta), v_{n}(\alpha(\zeta)) d \zeta d s\right.
$$

and

$$
\left|v(t)-v_{n}(t)\right| \leq \frac{N}{1-\left(N L_{g}+N^{2} L_{k}\right)} \phi(t) \text { for all } t \in I
$$

The uniqueness of the function $v_{n}$ implies that if $t \in I_{n}$, then

$$
v_{n}(t)=v_{n+1}(t)=v_{n+2}(t)=\cdots .
$$

For $t \in \mathbb{R}$, define $n(t) \in \mathbb{N}$ as

$$
n(t)=\min \left\{n \in \mathbb{N} \mid t \in I_{n}\right\} .
$$

Next, we define a function $v_{0}: \mathbb{R} \rightarrow \mathbb{R}$ by

$$
v_{0}(t)=v_{n(t)}(t)
$$

and claim that $v_{0}$ is continuous. For any $t_{1} \in \mathbb{R}$ we take the integer $n_{1}=n\left(t_{1}\right)$. Then, $t_{1}$ belongs to the interior of the interval $I_{n_{1}+1}$ and there exists positive $\varepsilon>0$ such that $v_{0}(t)=v_{n_{1}+1}(t)$ for all $t$ with $t_{1}-\varepsilon<t<t_{1}+\varepsilon$. Since $v_{n_{1}+1}$ is continuous at $t_{1}, v_{0}$ is continuous at $t_{1}$ for $t_{1} \in \mathbb{R}$.

Now, we prove that the continuous function $v_{0}$ satisfies $(2.5)$ and (2.7) for all $t \in \mathbb{R}$. Assume that $n(t)$ is an integer for any $t \in \mathbb{R}$. Then, by the use of (2.12) and (2.15), we 
have $t \in I_{n(t)}$ and

$$
\begin{aligned}
v_{0}(t)=v_{n(t)}(t) & =v(0)+\int_{0}^{t} g\left(\zeta, v_{n}(\zeta), v_{n}(\alpha(\zeta))\right) d \zeta \\
& +\int_{0}^{t} \int_{0}^{s} k\left(t, \zeta, v_{n}(\zeta), v_{n}(\alpha(\zeta)) d \zeta d s\right. \\
& =v(0)+\int_{0}^{t} g\left(\zeta, v_{0}(\zeta), v_{0}(\alpha(\zeta))\right) d \zeta \\
& +\int_{0}^{t} \int_{0}^{s} k\left(t, \zeta, v_{0}(\zeta), v_{0}(\alpha(\zeta))\right) d \zeta d s
\end{aligned}
$$

where the last equality holds true because $n(\zeta) \leq n(t)$ for all $\zeta \in I_{n(t)}$ and from equations (2.14) and (2.15) we get that

$$
v_{n(t)}(\zeta)=v_{n(\zeta)}(\zeta)=v_{0}(\zeta)
$$

Since $t \in I_{n(t)}$ for all $t \in \mathbb{R}$, so from (2.13) and (2.15), we have

$$
\left|v(t)-v_{0}(t)\right| \leq\left|v(t)-v_{n(t)}(t)\right| \leq \frac{N}{1-\left(N L_{g}+N^{2} L_{k}\right)} \phi(t),
$$

for any $t \in \mathbb{R}$.

Finally, we are going to prove that $v_{0}$ is unique. To do this we consider another continuous function $u_{0}: \mathbb{R} \rightarrow \mathbb{R}$ which satisfies (2.5) and (2.7), with $u_{0}$ instead of $v_{0}$, for all $t \in \mathbb{R}$. Let $t \in \mathbb{R}$ be an arbitrary number. Since the restrictions $\left.v_{0}\right|_{I_{n(t)}}$ and $\left.u_{0}\right|_{I_{n(t)}}$ satisfies (2.5) and (2.7) for each $t \in I_{n(t)}$, the uniqueness of $v_{n}(t)=\left.v_{0}\right|_{I_{n(t)}}$ suggest that

$$
v_{0}(t)=\left.v_{0}\right|_{I_{n(t)}}(t)=\left.u_{0}\right|_{I_{n(t)}}(t)=u_{0}(t) .
$$

We can prove similarly for the cases $I=(-\infty, T]$ and $I=[0, \infty)$.

\section{Hyers-Ulam stability}

In this section, we prove the Hyers-Ulam stability of the nonlinear Volterra integrodifferential equation with delay (1.1).

3.1. Theorem. Let $I=[0, T]$ be a non-degenerated interval, $L_{g}$ and $L_{k}$ be nonnegative constants such that $0<T L_{g}+\frac{T^{2}}{2} L_{k}<1$. Assume that $g: I \times \mathbb{R} \times \mathbb{R} \rightarrow \mathbb{R}$ is continuous function which satisfies the Lipschitz condition (2.1) and $k: I \times I \times \mathbb{R} \times \mathbb{R} \rightarrow \mathbb{R}$ is a continuous function which satisfies the Lipchitz condition (2.2). If for some $\sigma \geq 0$ and a continuously differentiable function $v: I \rightarrow \mathbb{R}$ we have

$$
\left|v^{\prime}(t)-g(t, v(t), v(\alpha(t)))-\int_{0}^{t} k(t, s, v(s), v(\alpha(s))) d s\right| \leq \sigma \text { for all } t \in I,
$$

then there exists a unique continuous function $v_{0}: I \rightarrow \mathbb{R}$ satisfying the equation (2.5) and

$$
\left|v(t)-v_{0}(t)\right| \leq \frac{T}{1-\left(T L_{g}+\frac{T^{2}}{2} L_{k}\right)} \sigma, \text { for all } t \in I .
$$

Proof. Let $Y$ be the set of all real valued continuous functions on closed and bounded interval $I$. For all $u, w \in Y$, we define a metric on $Y$ by

$$
d(u, w)=\inf \{K \in[0, \infty]:|u(t)-w(t)| \leq K \text { for all } t \in I\} .
$$

The metric space $(Y, d)$ is a complete generalized metric space, see [10]. Consider the operator $\Theta: Y \rightarrow Y$ defined by

$$
(\Theta u)(t)=v(0)+\int_{0}^{t} g(\zeta, u(\zeta), u(\alpha(\zeta))) d \zeta+\int_{0}^{t} \int_{0}^{s} k(t, \zeta, u(\zeta), u(\alpha(\zeta))) d \zeta d s
$$


for all $t \in I$ and for all $u \in Y$. Next, we need to check that the operator $\Theta$ is strictly contractive on the set $Y$. Suppose that $K_{u w} \in[0, \infty]$ be a constant with $d(u, w) \leq K_{u w}$ for any $u, w \in Y$. We have,

$$
|u(t)-w(t)| \leq K_{u w} \text {, for all } t \in I .
$$

By making the use of (2.1), (2.2), (3.4) and (3.5), we get

$$
\begin{aligned}
|(\Theta u)(t)-(\Theta w)(t)| & =\mid \int_{0}^{t}\{g(\zeta, u(\zeta), u(\alpha(\zeta)))-g(\zeta, w(\zeta), w(\alpha(\zeta)))\} d \zeta \\
& +\int_{0}^{t} \int_{0}^{s}\{k(t, \zeta, u(\zeta), u(\alpha(\zeta)))-k(t, \zeta, w(\zeta), w(\alpha(\zeta)))\} d \zeta d s \mid \\
& \leq \int_{0}^{t}|g(\zeta, u(\zeta), u(\alpha(\zeta)))-g(\zeta, w(\zeta), w(\alpha(\zeta)))| d \zeta \\
& +\int_{0}^{t} \int_{0}^{s}|k(t, \zeta, u(\zeta), u(\alpha(\zeta)))-k(t, \zeta, w(\zeta), w(\alpha(\zeta)))| d \zeta d s \\
& \leq L_{g} \int_{0}^{t}|u(\zeta)-w(\zeta)| d \zeta+L_{k} \int_{0}^{t} \int_{0}^{s}|u(\zeta)-w(\zeta)| d \zeta d s \\
& \leq L_{g} K_{u w} T+L_{k} K_{u w} \frac{T^{2}}{2} \\
& \leq K_{u w}\left(T L_{g}+\frac{T^{2}}{2} L_{k}\right), \text { for all } t \in I,
\end{aligned}
$$

i.e., $d(\Theta u, \Theta w) \leq K_{u w}\left(T L_{g}+\frac{T^{2}}{2} L_{k}\right)$. Hence, we may conclude that $d(\Theta u, \Theta w) \leq\left(T L_{g}+\frac{T^{2}}{2} L_{k}\right) d(u, w)$ for any $u, w \in Y$, where $0<T L_{g}+\frac{T^{2}}{2} L_{k}<1$. Suppose $w_{0} \in Y$ be arbitrary, there exists a constant $K \in[0, \infty]$ with

$$
\begin{aligned}
\left|\Theta w_{0}(t)-w_{0}(t)\right| & =\mid v(0)+\int_{0}^{t} g\left(\zeta, w_{0}(\zeta), w_{0}(\alpha(\zeta))\right) d \zeta \\
& +\int_{0}^{t} \int_{0}^{s} k\left(t, \zeta, u_{0}(\zeta), u_{0}(\alpha(\zeta))\right) d \zeta d s-w_{0} \mid \\
& \leq K, \text { for all } t \in I .
\end{aligned}
$$

Since $g\left(\zeta, w_{0}(\zeta), w_{0}(\alpha(\zeta))\right), k\left(t, \zeta, u_{0}(\zeta), u_{0}(\alpha(\zeta))\right)$ and $w_{0}$ are bounded. Thus, equation (3.3) implies that

$$
d\left(\Theta w_{0}, w_{0}\right)<\infty .
$$

So, according to Theorem 1.3, there exists a continuous function $v_{0}: I \rightarrow \mathbb{R}$ in a way that $\Theta^{n} w_{0} \rightarrow v_{0}$ in $(Y, d)$ and $\Theta v_{0}=v_{0}$, i.e., $v_{0}$ satisfies $(2.5)$ for all $t \in I$. As in the proof of Theorem 2.1, it can be verify easily that $\left\{w \in Y \mid d\left(w_{0}, w\right)<\infty\right\}$ is equal to $Y$. From Theorem 1.3, we conclude that $v_{0}$, given by equation (2.5), is the unique continuous function. Again from equation (2.3), we get

$$
-\sigma \leq v^{\prime}(t)-g(t, v(t), v(\alpha(t)))-\int_{0}^{t} k(t, s, v(s), v(\alpha(s))) d s \leq \sigma,
$$

for all $t \in I$. By integrating each term of inequality (3.6) from 0 to $t$, then we get

$$
\begin{aligned}
& \left|v(t)-v(0)-\int_{0}^{t} g\left(\zeta, v_{0}(\zeta), v_{0}(\alpha(\zeta))\right) d \zeta-\int_{0}^{t} \int_{0}^{s} k\left(t, \zeta, v_{0}(\zeta), v_{0}(\alpha(\zeta))\right) d \zeta d s\right| \\
& \leq \sigma T
\end{aligned}
$$


for all $t \in I$. So, it satisfies that $|v-\Theta v| \leq \sigma T$. Finally, Theorem 1.3 together with (2.11) implies that

$$
d\left(v, v_{0}\right) \leq \frac{T}{1-\left(T L_{g}+\frac{T^{2}}{2} L_{k}\right)} d(\Theta v, v) \leq \frac{T}{1-\left(T L_{g}+\frac{T^{2}}{2} L_{k}\right)} \sigma
$$

Now we present two examples which indicate how our theorems can be applied to concrete problems.

3.2. Example. Let $a>1, q \in(0, \infty)$ and $p$ are arbitrary but fixed constants. Consider the class of equations

$$
u^{\prime}(t)=g(t)+\frac{1}{2} \int_{0}^{t} \lambda(t-s)^{p}(u(s)+u(\alpha(s)+f(s)) d s, t \in[0, T]
$$

for $\lambda<\frac{(q \ln a)^{2}}{T^{p}}$, where $f(t)$ and $g(t)$ are any continuous functions. Here

$$
k(t, s, u(s), u(\alpha(s)))=\frac{1}{2} \lambda(t-s)^{p}(u(s)+u(\alpha(s))+f(s)) .
$$

Clearly

$$
\begin{aligned}
& \left|k\left(t, s, u_{1}(s), u_{1}(\alpha(s))\right)-k\left(t, s, u_{2}(s), u_{2}(\alpha(s))\right)\right| \\
& \leq \frac{1}{2} \lambda\left|(t-s)^{p}\right|\left(\left|u_{1}(s)-u_{2}(s)\right|+\mid u_{1}\left(\alpha(s)-u_{2}(\alpha(s)) \mid\right)\right) \\
& \leq \lambda T^{p}\left\|u_{1}-u_{2}\right\| .
\end{aligned}
$$

Let $v: I \rightarrow R$ be such that

$$
\left|v^{\prime}(t)-g(t)-\frac{1}{2} \int_{0}^{t}\left(\lambda(t-s)^{p}(v(s)+v(\alpha(s))+f(s))\right) d s\right| \leq \sigma(t)=a^{q t}, t \in[0, T] .
$$

Clearly,

$$
\left|\int_{0}^{t} \sigma(t) d t\right|=\left|\int_{0}^{t} a^{q t} d t\right|=\frac{1}{q \ln a} a^{q t}=\frac{1}{q \ln a} \sigma(t)
$$

for all $t \in[0, T]$. Theorem 2.1 ensures the existence of a unique continuous function $v: I \rightarrow R$ that solves (3.7) and

$$
\left|v(t)-v_{0}(t)\right| \leq \frac{q \ln a}{(q \ln a)^{2}-T^{p} \lambda} a^{q t}, t \in[0, T] .
$$

3.3. Example. Consider the above class of problems for $\lambda<\frac{2}{T^{p+2}}$. Let for some $\sigma>0$ and $v: I \rightarrow R$ we have

$$
\left|v^{\prime}(t)-g(t)+\frac{1}{2} \int_{0}^{t} \lambda(t-s)^{p}(v(s)+v(\alpha(s))+f(s)) d s\right| \leq \sigma, t \in[0, T] .
$$

In the light of Theorem 3.1, there exists a unique continuous function $v: I \rightarrow R$ that solves (3.7) for $\lambda<\frac{2}{T^{p+2}}$ and

$$
\left|v(t)-v_{0}(t)\right| \leq \frac{2 T}{2-T^{p+2} \lambda} \sigma, t \in[0, T] .
$$

Conclusion. In this manuscript, we investigated the Hyers-Ulam-Rassias stability and Hyers-Ulam stability of a nonlinear Volterra integrodifferential equation with delay by using a fixed point method. 


\section{References}

[1] M. Akkouchi, Hyers-Ulam-Rassias stability of nonlinear Volterra integral equations via a fixed point approach, Acta Univ. Apulensis Math. Inform., 26 (2011), 257-266.

[2] L. Cădariu and V. Radu, On the stability of the Cauchy functional equation: a fixed point approach, Grazer Math. Ber., 346 (2004), 43-52.

[3] L. P. Castro and A. Ramos, Hyers-Ulam-Rassias stability for a class of nonlinear Volterra integral equations, Banach J. Math. Anal., 3 (2009), 36-43.

[4] L. P. Castro, A. Ramos, Hyers-Ulam and Hyers-Ulam-Rassias stability of Volterra integral equations with delay, Integral methods in science and engineering, Birkhauser Boston, Inc., Boston, MA, 1 (2010), 85-94.

[5] J. B. Diaz and B. Margolis, A fixed point theorem of the alternative, for contractions on a generalized complete metric space, Bull. Amer. Math. Soc., 74 (1968), 305-309.

[6] M. Gachpazan and O. Baghani, Hyers-Ulam stability of Volterra integral equation, J. Nonl. Anal. Appl., 1 (2010), 19-25.

[7] M. Gachpazan and O. Baghani, Hyers-Ulam stability of nonlinear integral equation, Fix. P. Theo. Appl., 2010 (2010), 6 pages.

[8] D. H. Hyers, On the stability of the linear functional equation, Proc. Nat. Acad. Sci. U. S. A, 27 (1941), 222-224.

[9] S. M. Jung, A fixed point approach to the stability of a Volterra integral equation. Fix. P. Theo. Appl., 2007 (2007), 9 pages.

[10] S. M. Jung, A fixed point approach to the stability of differential equations $y^{\prime}=F(x, y)$, Bull. Malays. Math. Sci. Soc., 33 (2010), 47-56.

[11] S. M. Jung, S. Sevgin and H. Sevli, On the perturbation of Volterra integro-differential equations, Appl. Math. Lett., 26 (2013), 665-669.

[12] J. R. Morales and E. M. Rojas, Hyers-Ulam and Hyers-Ulam-Rassias stability of nonlinear integral equations with delay, Int. J. Nonl. Anal. Appl., 2 (2011), 1-6.

[13] T. M. Rassias, On the stability of the linear mapping in Banach spaces, Proc. Amer. Math. Soc., 72 (1978), 297-300.

[14] J. M. Rassias and M. Eslamian, Fixed points and stability of nonic functional equation in quasi- $\beta$-normed spaces, Cont. Anal. Appl. Math., 3 (2015), 293-309.

[15] S. M. Ulam, Problems in Modern Mathematics, Science Editions John Wiley \& Sons, New York, (1960).

[16] A. Zada, R. Shah and T. Li, Integral type contraction and coupled coincidence fixed point theorems for two pairs in G-metric spaces, Hacet. J. Math. Stat., 45 (2016), 1475-1484. 